\title{
A Rare Case of Bilateral Malignant Brenner Tumors of the Ovaries Associated with Peritoneal Effusion
}

Jianing Tong ( $\square$ 15036692727@139.com )

Shenzhen Maternity and Healthcare Hospital

Jianmin Niu

Shenzhen Maternity and Healthcare Hospital

Qiaoyun Li

The Fifth Affiliated Hospital of Zhengzhou University

Li Hu

The Fifth Affiliated Hospital of Zhengzhou University

Hui Zhang

The Fifth Affiliated hospital of Zhenzghou University

\section{Case report}

Keywords: Malignant Brenner Tumor, Ovarian Tumor

Posted Date: October 26th, 2020

DOI: https://doi.org/10.21203/rs.3.rs-96101/v1

License: (c) (1) This work is licensed under a Creative Commons Attribution 4.0 International License. Read Full License 


\title{
A Rare Case of Bilateral Malignant Brenner Tumors of the Ovaries Associated with Peritoneal Effusion
}

Jianing Tong ${ }^{1}$; Jianmin $\mathrm{Niu}^{1} ;$ Qiaoyun $\mathrm{li}^{2} ; \mathrm{Li} \mathrm{Hu}^{2} ;$ Hui Zhang $^{2}$

Authors and affiliations:

1:Shenzhen Maternity and Child Healthcare Hospital, Southern Medical University, Shenzhen, Guangdong Province, China.

2:The Fifth Affiliated Hospital of Zhengzhou University,Zhengzhou,Henan Province, China.

Correspondence to:

Dr. Jianing Tong, $\mathrm{PhD}$

Shenzhen Maternity and Child Healthcare Hospital, Southern Medical University, Shenzhen, Guangdong province, China

Email:15036692727@139.com

\begin{abstract}
Background:Malignant ovarian Brenner tumors are extremely rare worldwide, accounting for only $1 \%$ of malignant ovarian tumors. Their clinical symptoms and pathology are complex and erratic,and the images are mostly non-specific. This poses difficulties in preoperative diagnosis and distinguishing them from other cystic solid ovarian tumors.Here this study has reported on a case of bilateral malignant ovarian Brenner tumor with peritoneal effusion.
\end{abstract}


Case presentation: A 54-year-old woman presented with intermittent vaginal bleeding for more than two months and abdominal pain for one month. Two hard mass of five centimeters with poor movement could be touched at each side of the ovarian areas. The CT findings indicated the presence of two large cystic and solid masses in both adnexal regions. The operation had watched the size of the masses in the bilateral ovarian was both $6 \mathrm{~cm} \times 6 \mathrm{~cm} \times 5 \mathrm{~cm}$ and their surface ulceration showed rotten fleshy tissue. After the operation,combined with morphological and immunohistochemical features, the ovarian specimens were consistent with bilateral ovarian malignant Brenner tumors.

Conclusions: Although the incidence of bilateral malignant OBT is extremely low and this disease is extremely rare clinically, the gynecologists should be more informed of its diagnosis and treatment.

[Keywords]Malignant Brenner Tumor; Ovarian Tumor

\section{Background}

Ovarian Brenner Tumors (OBT) are the rarest ovarian epithelial tumors that account for only $1.5 \%$ of all ovarian tumors. Most of them are benign (95\%-97\%), with the borderline and malignant ones only accounting for 3\%-5\% ${ }^{[1]}$. Malignant OBTs(MBTs) are very rare, and less than 200 cases have been reported to date, which only constitutes one percent of all the ovarian malignant tumors ${ }^{[2-3]}$. Their clinical symptoms and pathology are complex and erratic,and the images are mostly non-specific. This poses difficulties in preoperative diagnosis and distinguishing them from other cystic solid ovarian tumors ${ }^{[4]}$. Moreover,no standard method of diagnosis and treatment exit to date. Additionally, peritoneal effusion is quite rare in MBTs and is only found in $10 \%$ of the patients ${ }^{[5-7]}$. This study has reported on a case of bilateral malignant OBT with peritoneal effusion.

\section{Case Report}


A 54-year-old woman presented with intermittent vaginal bleeding for more than two months and abdominal pain for one month. She came to the hospital on June $17^{\text {th }}$, 2017. The patient had an appendectomy 26 years ago and went through natural menopause three years ago.On performing the admission examination, the following observations were made: there were no enlarged superficial lymph nodes in the whole body but a hard mass with unclear size could be touched in the upper abdomen. The upper abdomen had positive tenderness accompanied by obvious percussion pain in the liver area. The gynecological examination was then performed and the following were observed: the vagina was smooth with a large amount of bloody fluid and its dome was not thickened. A hyperplastic tumor with a three-centimeter diameter resembling cauliflower was found in the cervical canal and active bleeding was observed. The uterine body had positive tenderness with poor movement and the ligaments were not thickened at both sides of the uterus. Two hard mass of five centimeters with poor movement could be touched at each side of the ovarian areas. Next,the trimanual gynecologic examination was carried and it was observed that the sacral ligaments and the main ligaments were not thickened; there was no abnormal mass in the rectouterine fossa; the rectal mucosa was smooth, and the finger covers were not stained with blood. The tumor markers of CA125, CEA, and AFP were 213 $\mathrm{KU} / \mathrm{L}$, CEA $3.60 \mathrm{KU} / \mathrm{L}$, and $1.31 \mu \mathrm{g} / \mathrm{L}$, respectively. The CT findings indicated the presence of two large cystic and solid masses in both adnexal regions, and that the solid components were enhanced unevenly. The cervix was irregular in shape and showed uneven and obvious enhancement. The endometrium was inhomogeneously thickened. Multiple small nodules were observed behind the peritoneum and a small amount of fluid density was observed in the pelvic cavity (Picture 1). 


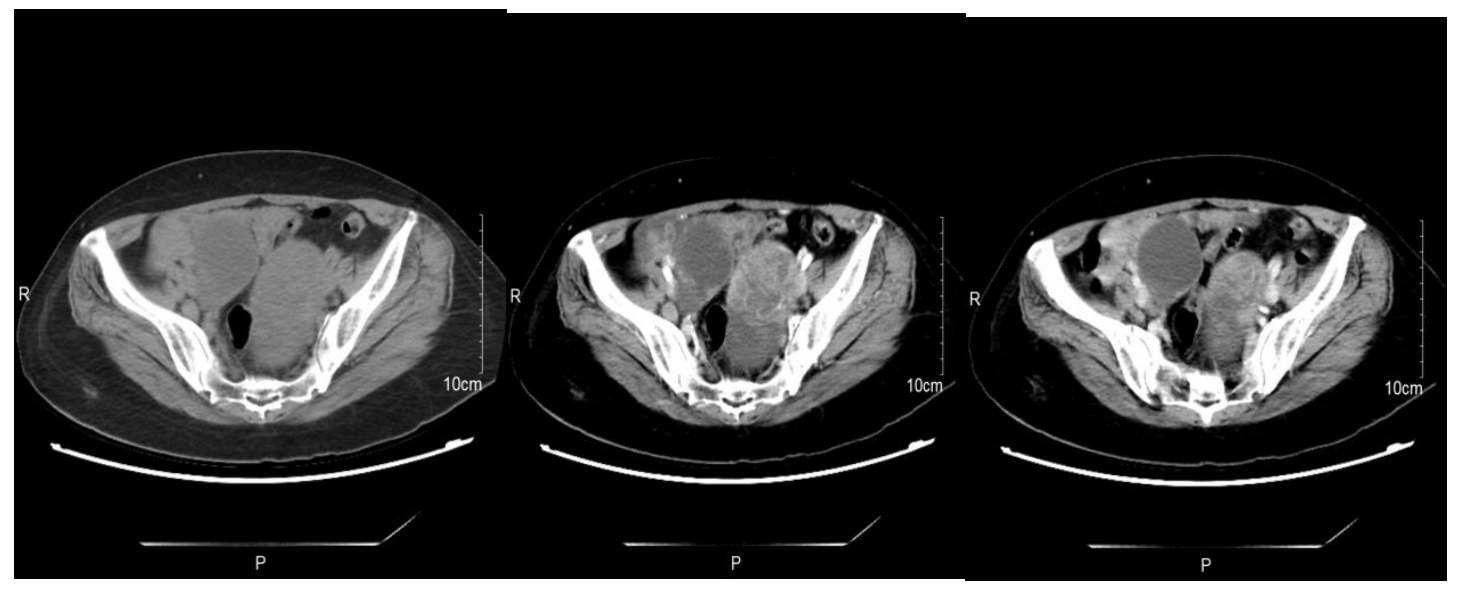

Arterial phase

Diagram of plain

venous phase diagram

Picture 1:Abdominal CT of the patient (Enhanced CT scan of the whole abdomen, arterial phase, venous phase)

Diagnosis of CT:1. Bilateral adnexal cystic and the solid mass-the possibility of ovarian cystadenocarcinoma. 2. The irregular shape of the cervix. 3. Small amounts of pelvic fluid. 4. Multiple small retroperitoneal lymph nodes.

Therefore, the biopsy of cervical canal vegetation under the colposcopy was performed. The results showed that it was a moderately-poorly differentiated squamous cell carcinoma(Picture 2).Therefore, the preliminary diagnosis suspected one among cervical cancer, endometrial cancer, and ovarian tumor. The preoperative discussion in the department considered the possibility of endometrial cancer and its invasion to the cervix, which indicated the patient had surgical indications. Therefore, extensive hysterectomy, bilateral appendectomy, and lymphadenectomy were to be performed. Finally, on $23^{\text {rd }}$ June,ovarian cancer tumor cytoreductive surgery and intestinal adhesion lysis under the general anesthesia combined epidural anesthesia were performed on the patient. 


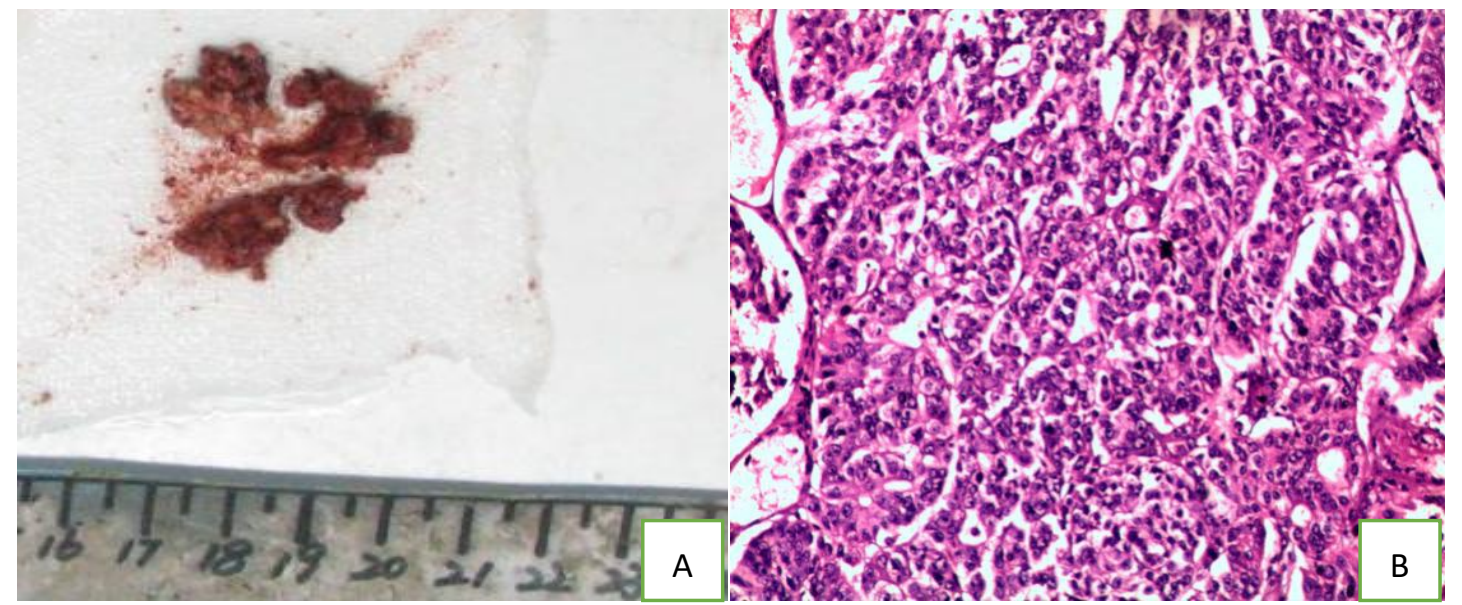

Picture 2:Poorly differentiated squamous cell carcinomam of the cervix.

During the operation, about $500 \mathrm{ml}$ of bloody ascites was found and $200 \mathrm{ml}$ was taken for cytological examination. The omentum majus was thickened to form an $8 \mathrm{~cm} \mathrm{x}$ $8 \mathrm{~cm}$ hard cake(Picture 3). The surface of the pelvic peritoneum, colonic groove, right colon bowel, and bladder were all covered with gray brittle nodules and the gastric surface was covered with grain size nodules. The surfaces of the liver and the transverse septum were smooth. The uterus was of two-months pregnancy size and there were several peanut-sized nodules in the isthmus. There was no swollen lymph node in the pelvic cavity and beside the abdominal aorta. The bilateral ovarian was enlarged to $6 \mathrm{~cm} \times 6 \mathrm{~cm} \times 5 \mathrm{~cm}$ size with surface ulceration showing rotten fleshy tissue. The bilateral fallopian tubes were thickened and the umbrella section of the right fallopian tube presented rotten fleshy tissue. The bilateral adnexa adhered closely to the lateral pelvic wall and intestinal canal(Picture 4). 


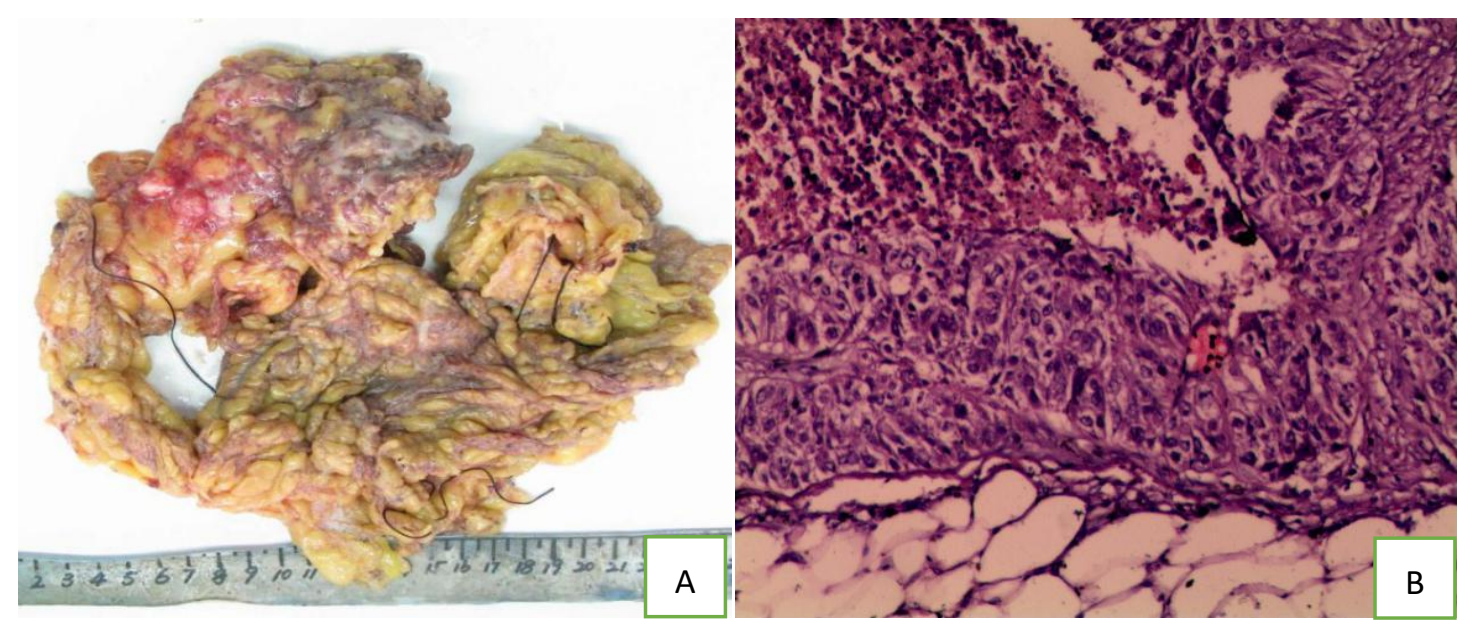

Picture 3:The omentum majus thickening to form an $8 \mathrm{~cm} \times 8 \mathrm{~cm}$ hard cake.Multifocal, implantable, carcinoma nodules of the omentum majus

The cytological examination of ascites reported the presence of epithelial cells which were slightly atypical and were considered to have originated from the pelvic malignant tumor. Therefore, the intestinal adhesion was separated, the arteries and the veins of the ovaries ligated, and then the whole uterus and double appendages were removed. After dissecting the uterus, a rotten flesh-like mass of $3 \mathrm{~cm} \times 4 \mathrm{~cm}$ size was found in the uterine cavity which is mainly concentrated in the isthmus and the right wall of the posterior wall of the uterus. The mass went down to the cervical canal, but not to the external orifice of the cervical canal(Picture 4).

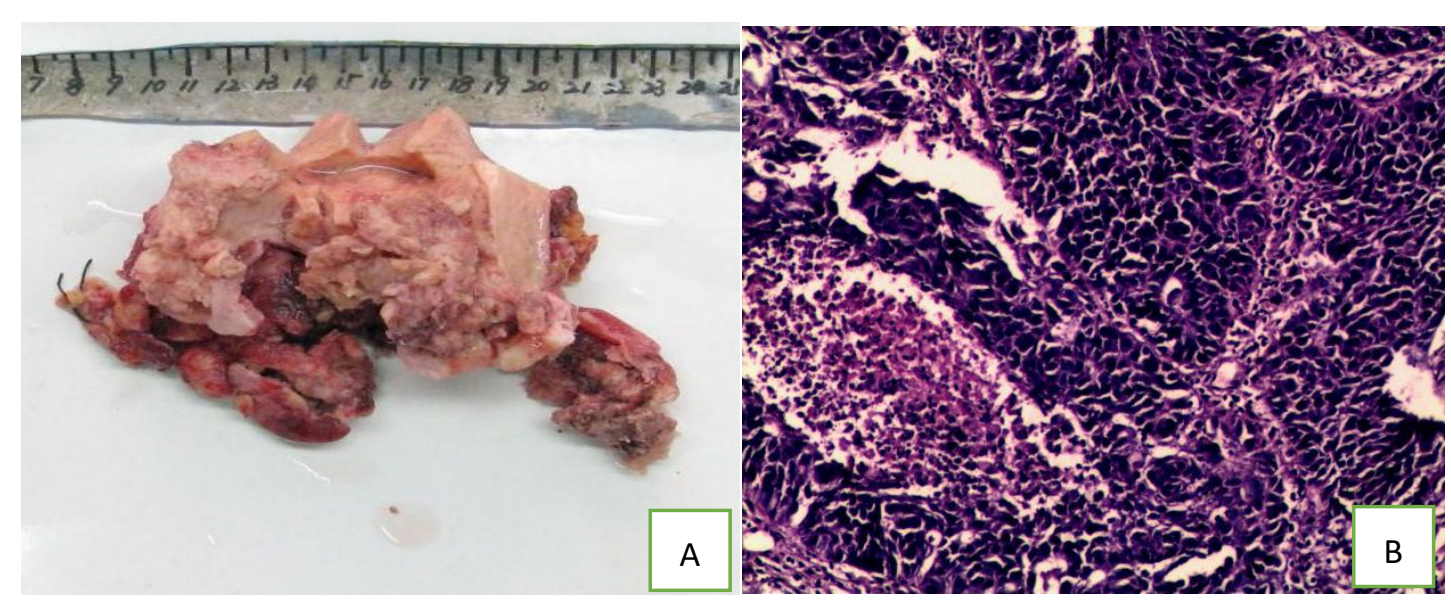


A: Bilateral malignant Brenner ovarian tumor extending to the entire outer Mesangium with multiple foci of necrosis and cystic degeneration.B: Both fallopian tubes and their mesentery are infiltrative, with multiple nodules surrounding the double appendages. Carcinoma cells invading the uterine wall and reaching the uterine cavity and internal orifice. No invasive carcinoma found in the external orifice of the cervix.

Rapid freezing pathology showed that both ovaries seemed to have malignant transitional cell carcinoma,which invaded the whole layer with necrotic cystic degeneration. Multiple cancerous nodules were found on the surface of both ovarian mesenteries and mesosalpinx. At the same time, the transitional cell carcinoma also invaded the whole layer of the uterus, reaching the uterine cavity and the internal orifice of the cervix.

From the above findings, stage III of ovarian cancer was diagnosed during operation, and therefore tumor cytoreductive surgery was performed. The cancer nodules on the surface of the intestine and the peritoneum were removed, followed by removal of the omentum majus. Because the pelvic cavity was hyperemic with extensive adhesions, the lymph node dissection was not performed.

\section{Final Pathological Diagnosis after the Operation}

\subsection{Microscopy}

Combined with morphological and immunohistochemical features, the ovarian specimens were consistent with malignant bilateral ovarian Brenner tumors.The invasion of carcinoma was spread into the whole layer with multiple focal necroses. The invasion into the bilateral fallopian tubes and mesangial membranes with 
multiple cancer nodules around bilateral adnexa was also observed. The carcinoma cells invaded the uterine wall all the layer up to the uterine cavity and the intrauterine orifice, and no cancer invasion was found in the external cervical orifice. The adenocarcinoma of the right half colon and abdominal neoplasm which both originated from the ovaries infiltrated and metastasized to the entire intestinal wall was also observed. Formed carcinoma nodules can be seen in the per-intestinal nodules and also in the omentum majus. The samples taken from pelvic and para-aortic lymph nodes were both negative.

The morphology of bilateral ovarian tumors was similar to those tumor cells which showed cystic-solid distribution, and some cells were adenoid that suggested the mutual transformation and migration among typical benign, borderline and malignant Brenner tumor components. Benign and borderline cell morphology can be seen in malignant Brenner tumor with the prominent heteromorphism of the cells. Moreover, the cells were non-polarity,their mitotic and pathological mitotic images were commonly observed. Morphologically, the tumors belonged to transitional epithelial carcinoma which was mainly composed of epithelial cell nests and fibrous connective tissue stroma. The tumor cells were polygonal with abundant cytoplasm and the nucleus was oval. The longitudinal sulcus of the nucleus was visible and infiltrated into the stroma to complete the metastasis to the surrounding tissues and organs(Picture 2B-3B-4B).

\subsection{Immunohistochemical phenotype}

The tumor cells were mostly positive for CK5/6, CK34ßE12, P63 and CK20, as well as CA125、Pgp and P53. The proliferation index of PR and ER was about 95\% and $80 \%$, respectively. The positive rate of ki-67 was approximately $80 \%$. 


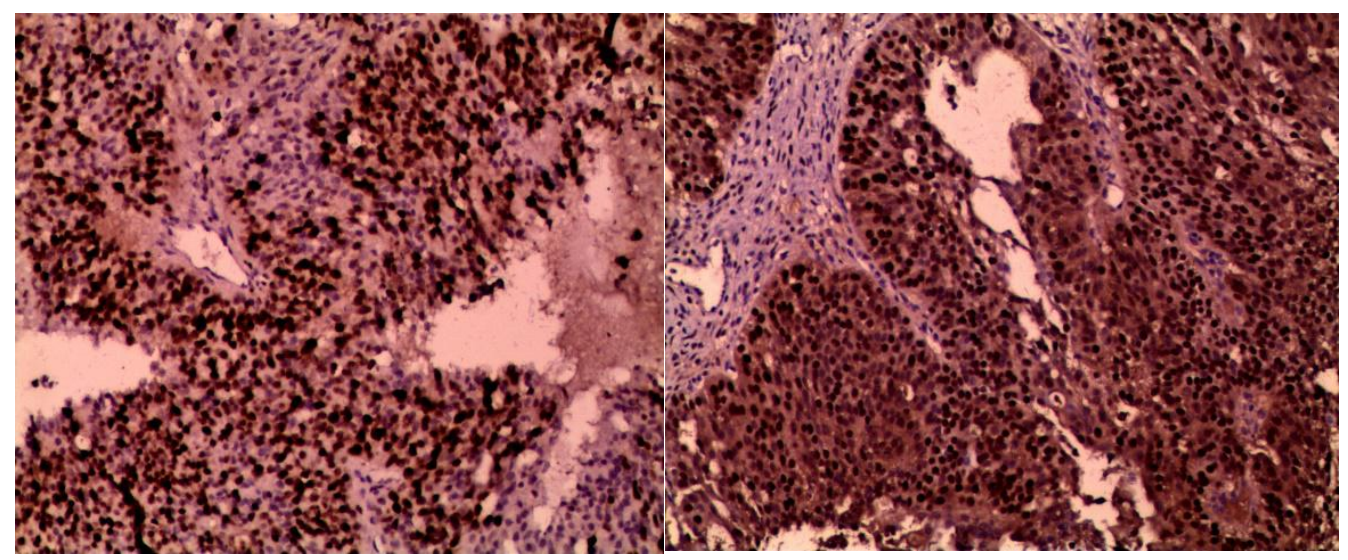

Picture 5 Histopathological characteristics of the specimen by HE staining (HEx100)

IHC immunohistochemical results: CK5/6(multiple foci + ) , CK34 E12(multiple foci + ) , P63(multiple foci + ) , CK20(multiple foci +) , CA125(+) , PR (95\%) , ER (80\%) , PGP (++) , P53(++) , Ki-67(+ about 80\%) . Combined with $\mathrm{HE}$ staining, it was indicated to be malignant ovarian Brenner tumor, and the performance of most cells was corresponded with transitional cell carcinoma, the performance of a small number with squamous cell carcinoma and undifferentiated carcinoma with high proliferative activity.

\subsection{The Treatment Process}

After the operation, the final diagnoses were Stage III C of bilateral ovarian malignant Brenner tumor and moderately-poorly differentiated squamous cell carcinoma of the cervix. Thus, intravenous chemotherapy of paclitaxel $270 \mathrm{mg}$ and carboplatin $0.5 \mathrm{~g}$ was initiated on $29^{\text {th }}$ and $30^{\text {th }}$ June 2017 ,respectively. The process went well and from $27^{\text {th }}$ to $28^{\text {th }}$ July, the patient was continued to be given intravenous chemotherapy of paclitaxel $270 \mathrm{mg}$ plus carboplatin $0.5 \mathrm{~g}$.During this process, she had nausea and low white blood cell response that were relieved by the treatment of acid suppression, antiemetic, and leukocyte rising. From $17^{\text {th }}$ to $18^{\text {th }}$ August, paclitaxel 270mg plus cisplatin $100 \mathrm{mg}$ was administered intravenously. At the same time, the patient received acid inhibition and anti-vomiting treatments.

On September $6^{\text {th }}$, the CA125 decreased to $157.1 \mathrm{KU} / \mathrm{L}$. The CT scan of the full abdomen showed that the uterus was absent and there were no obvious enlarged 
lymph nodes in the abdominal cavity. Paclitaxel $270 \mathrm{mg}$ was then administered on $11^{\text {th }}$ September,and cisplatin100mg supplemented with antiemetic and liver protection drugs was used on $12^{\text {th }}$ September.From $4^{\text {th }}$ to $5^{\text {th }}$ October, intravenous chemotherapy of paclitaxel 270mg and cisplatin 100mg was administered again.During the chemotherapy, the patient developed nausea and low leukocyte response which were relieved by acid suppression, antiemetic, and leukocytic treatment. Because the patient could not tolerate the digestive reaction of cisplatin, it was replaced with intravenous chemotherapy of nedaplatin $120 \mathrm{mg}$ and docetaxel $120 \mathrm{mg}$ on October $31^{\text {st }}$.She did not complain of discomfort any more during the process. On reexamination at the end of the chemotherapy, the CA125 was $208.50 \mathrm{U} / \mathrm{ml}$, and CEA was $3.30 \mathrm{ng} / \mathrm{ml}$. Due to the late staging, radiotherapy was tried to perform four times (external irradiation $50 \mathrm{~Gy}$, conformal intensity modulation, 25 times) from $10^{\text {th }}$ November.

However, on December $13^{\text {th }}$, the patient presented with abdominal pain and decreased ventilation, and lack of excrement for the past three days. A palpable mass could be touched in the abdomen and a gas-liquid plane was found on the DR examination. The abdominal CT also revealed a section of small intestines dilatated and effused with the gas-liquid plane which indicated small intestinal obstruction. Even though the patient was given gastrointestinal decompression and fluid supplement treatment, she still complained of abdominal distension and abdominal pain. Further treatment by the care group was recommended, but the family refused and signed out of the hospital. Finally, the patient died nine months after the first operation due to uncorrected grade IV myelosuppression and pulmonary infection(Picture 6). 


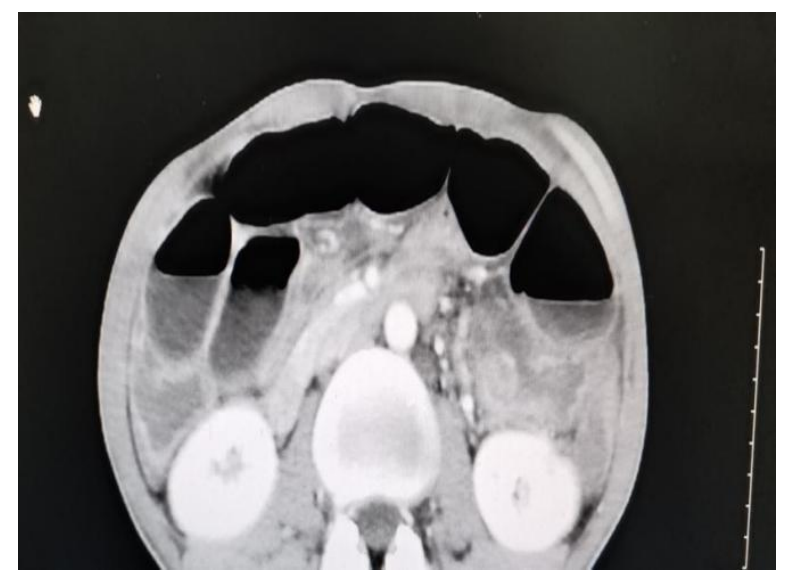

Picture 6 Abdominal CT of the patient (Enhanced CT scan of the whole abdomen, arterial phase, axial)

\section{Discussion}

\subsection{Clinical Symptoms}

Ovarian Brenner tumor (OBT) was first reported by MacNaughton-Jones in 1898 and named by Fritz Brenner in $1907^{[1]}$.It accounts for $2 \%-5 \%$ of all ovarian tumors, $95 \%$ of which are benign with no obvious symptoms ${ }^{[2]}$. The incidence of malignant OBT(MBT) is less than $1 \%$ and is commonly found in unilateral ovaries. The incidence of $12 \%$ was reported for bilateral ovaries and is more common in patients aged fifty to sixty. About half of these patients are found accidentally with no obvious symptoms and mostly in their middle or late stages. Most patients seek medical help with abdominal mass or digestive system symptoms as the first complaint ${ }^{[3-5]}$.

MBT is very rare with few local and global literature reports. Most of the documented literature are case reports which lack epidemiological and prognostic clinical data. The first two cases of malignant OBT were reported by Claés von Numers in $1945^{[2]}$.Some malignant cases had irregular vaginal bleeding,signifying the importance of routine gynecological examination for adult women ${ }^{[6]}$. In some cases, the level of estrogen was increased, and the metastatic foci presented the corresponding symptoms ${ }^{[7]}$. It was reported that four out of nine cases of MBT, 
presented with pleural effusion, and among the four cases, two were accompanied by ascites $^{[4]}$. Furthermore, it was considered that the presence of pleural effusion and ascites could be used as the reference indices for distinguishing benign and malignant OBT.

Therefore, special attention should be paid to the examination of the reproductive organs, such as the ovaries and the fallopian tubes to exclude the lesions when the elderly women have unexplained pleural effusion and ascites. A case of a 63-year-old woman with malignant OBT who was initially diagnosed with cervical cancer stage IA by preoperative loop electrosurgical excision had already been reported. In that case,preoperative abdominal ultrasound also found bilateral adnexal masses with a size of 50-60 mm. The masses were removed during the operation and sent to the rapid pathology which indicated that they were malignant Brenner tumor of the ovaries $^{[7]}$.

\subsection{Tumor Markers}

Currently, there are no specific tumor markers for malignant OBT. In 13 cases of malignant OBT, the preoperative serum CA125 of six patients $(46.2 \%)$ were higher than in normal people, and was significantly increased in two cases but decreased dramatically to normal levels after the chemotherapy. At present, it is believed that serum CA125 is still the preferred reference index for judging benign and malignant ovarian epithelial tumors including MBT. It is also used to predict the treatment effects and prognosis under certain conditions ${ }^{[8-11]}$.

\subsection{Pathological Origins}

There are different opinions on the origin of MBT in the ovary. Some believe that it is derived from the germinal epithelium on the surface of the ovary. Others think that it 
is originated from the Walthard cells, the ovarian net, teratoma, or the ovarian stroma. Walthard cell nest is formed by metaplastic transitional epithelium and trapped in the tissues adjacent to the fallopian tubes, where most Brenner tumors are located. From the perspective of embryology, most scholars believe that the ovarian MBT is derived from the surface epithelium, which is generated by the the epithelium ${ }^{[12-13]}$.

In 2003, $\mathrm{WHO}^{[5]}$ classified $\mathrm{OBT}$ as ovarian surface epithelial-stromal tumor and defined it as ovarian tumors that are histologically similar to the urothelium and its tumors. The OBT includes benign, borderline, malignant OBT, and transitional cell

carcinoma. The histological characteristics of OBT are very similar to those of non-keratinizing squamous cell carcinoma and transitional cell carcinoma. The benign Brenner cell nests can be seen in the tumor, which differentiates OBT from transitional cell carcinoma ${ }^{[7]}$. According to the immunohistochemical markers, OBTs present the characteristics of urethral epithelial differentiation, has similar immunophenotype to that of normal urethral epithelium and urethral epithelial tumor, such as CK7 and 13, and has positive expression of Gata3 and $\mathrm{S} 100 \mathrm{P}{ }^{[8]}$.

\subsection{Pathological Features}

The extreme rarity of the malignant Brenner tumors can be explained by the following proofs $^{[4,12-13]}$. The epithelial component must have obvious atypia histologically; the transitional morphology between benign and malignant tumors must be found; malignant epithelial components must infiltrate the interstitium; the mucous cystadenocarcinoma must be distinguished from the malignant transformation of Brenner tumor into mucinous cystadenocarcinoma and the average age of the patient is over 60 years old. The histological features of MBT are epithelial nests showing malignant cytological evidence which presents with increased epithelial layers. The nuclei are deeply stained with pleomorphic and obvious mitotic activity, which are characterized by pathological mitotic figures and occasionally giant tumor cells. 
Squamous areas or parts of the cells prone to glandular differentiation are frequently observed. The stroma is often sparse with inflammatory cells infiltrated to varying degrees. Necrosis is common in the tumors, and calcifications occasionally appear. Besides, irregular nests, branching and fusion of epithelial cells, interstitial deficiency due to the dense epithelial masses, and fibroproliferative interstitial response are all useful features for pathological diagnosis.

The diagnosis of MBT requires that its infiltrating elements be high-grade squamous-cell carcinoma which sometimes can be low-grade, closely packed, and irregular cells. At the same time, there must be a benign or proliferative transitional cell tumor adjacent to the tumor for the diagnosis of MBT otherwise, it should be diagnosed as ovarian transitional cell carcinoma. Without benign or proliferative transitional cell components, it is difficult to differentiate MBT from adenosquamous carcinoma and other poorly differentiated ovarian cancers. The presence of a clear mucinous adenocarcinoma component confirmed MBT whereas tumors from or adjacent to the endometriosis cyst were considered endometrioid tumor ${ }^{[14-15]}$.

Roma et $\mathrm{al}^{[9]}$ conducted a molecular analysis on 32 cases of Brenner tumors and found that $43 \%(13 / 32)$ had subserous Walthard cell nests in the serosa of fallopian tubes. Four cases of malignant Brenner tumors had obvious interstitial infiltration, and the components of benign or borderline Brenner tumors were visible. The tumor cells were obviously heteromorphic with large and deep stained nuclei. The mitotic figures of four cases of malignant Brenner tumor were similar to those of infiltrated urothelial carcinoma, adenocarcinoma, and undifferentiated carcinoma. Moreover, among the four cases, one presented in the fallopian tube and the omentum. In some areas, cancer cells were in the form of nests or cords and grew invasively in the proliferative interstitium of connective tissue. Cancer cells in some areas have special characteristics like irregular nests, budding, branching, fusion, and interstitial 
sclerosis or disappearance, and some cell nests initially appeared obviously to be epithelial metaplasia of mucinous.

Kondi-Pafiti et $\mathrm{al}^{[14]}$ studied 30 cases of ovarian Brenner tumors immunohistochemically and found that all cases expressed CK7, but did not express CK20,while WT-1 was focally positive in five cases. A casereported that p63 was positive in benign and borderline ovarian Brenner tumors, whereas p16 and p53 were negative in both types of tumors ${ }^{[13]}$.Malignant Brenner tumors need to be differentiated from metastatic transitional cell carcinoma, which is diagnosed based on the history of a tumor in the urinary system and the immunohistochemical markers. Both urinary transitional cell carcinomas and ovarian malignant Brenner tumors are usually CK7 positive, but only the former is CK20 positive ${ }^{[15]}$.

\subsection{Treatment and Prognosis}

Surgery is still the most effective treatment, and similar to the epithelial ovarian cancer, it is advisable to perform the comprehensive staging surgery for the MBT as much as possible. The staging can be done with pelvic and abdominal lymph node dissection; however, studies have shown that it has no improvement on the survival rate, and retroperitoneal lymph node dissection is not emphasized. Malignant patients should undergo complete hysterectomy with double adnexa as well as pelvic and abdominal lymph node dissection ${ }^{[11-12]}$.

The six courses of chemotherapy with paclitaxel plus platinum might be the main chemotherapeutic approach after surgery as the curative effect could be better. Systemic chemotherapy is required after surgery and was found to be effective for patients with local recurrence. Patients with malignant OBT confined to the ovary might have a good prognosis with a 5-year survival rate. When distant metastasis such 
as abdominal metastasis occurs, the opportunity of operation is lost and therefore, conservative treatment with a five-year survival rate of $51.3 \%$ would be the main treatment ${ }^{[13-14]}$.

The operative principle of malignant Brenner tumor is consistent with that of epithelial ovarian cancers, but lymphadenectomy could be of no benefit to the prognosis. The prognosis of those with extraovarian dissemination is extremely poor. The treatment regimen of malignant OBT remains unclear due to its rarity and lack of clinical application of the corresponding scale but generally, it is similar to that of ovarian epithelial malignant tumors. The standard regimen is paclitaxel plus platinum and although most malignant $\mathrm{OBT}$ are sensitive to chemotherapy, the recurrence rate is still high ${ }^{[15-16]}$.In another investigation, nine out of ten cases of malignant OBT treated with the chemotherapy of paclitaxel plus platinum were completely relieved, but the recurrence rate was as high as $52 \%{ }^{[6]}$.

In our case introduced above, the patient presented with extremely atypical symptoms with cervical lesions and ascites, which were once considered as "cervical tumor" before surgery. Furthermore, the patient was in poor condition with abdominal metastasis occurring before it was discovered. And due to the late stage and lack of the related experiences, the doctors had no other better option and did not make wise decisions, thus chemotherapy and radiotherapy were not so effective and so standard.This resulted in an extremely poor prognosis which is a sad lesson needs every medical practitioners to be warned.

\section{Conclusion}

In conclusion, although the incidence of bilateral malignant OBT is extremely low and this disease is extremely rare clinically, the gynecologists should be more 
informed of its diagnosis and treatment. Besides, the medical practitioners should advise the patients to have a regular physical examination, which might help in early diagnosis and treatment hence, prolonging the survival period.

\section{Abbreviations}

CK:Cytokeratin; CA125:Cancer Antigen125;Pgp:PGP Antibody

\section{Declarations}

\section{Ethics approval and consent to participate}

This study was approved by the program of Human Ethics Committee of the Health Administration of Henan Province(LHGJ20191494). A written informed consent was obtained from all the patients at the time of admission, with which the blood, tissue and other sample were authorized to scientific purpose.

\section{Consent for publication}

Written informed consent was obtained from the patient for the publication of this case report and any accompanying images. A copy of the written consent is available.

\section{Availability of data and materials}

The authors declare that all data and materials of the article are available to all readers of our article.

\section{Competing interests}

The authors declare that they have no competing interests.

\section{Funding}

The authors declare that there was no funding for this article.

\section{Authors' contributions}

JNT carried out the immunohistochemical staining and wrote the manuscript. JMN revised the manuscript for important intellectual content, and gave the final approval 
of the version to be published.QYL,LH and HZ helped collect the materials and information. All of the authors have read and approved the final manuscript.

\section{Acknowledgements}

Not applicable

\section{References}

[1] Brenner F. Das Oophoroma folliculare. Frankf Z Path. 1907; 1: 150-171.

[2] Susan M. Langa, Anne M. Mills,Leigh A. Cantrel Malignant Brenner tumor of the ovary: Review and case report.Gynecologic Oncology Reports. 2017, 22: 26-31.

[3] Austin, R.M., Norris, HJ. Malignant Brenner tumor and transitional cell carcinoma of the ovary: a comparison. Int J Gynecol Pathol.1987,6:29-39.

[4] Burg J, Kommoss F, Bittinger F, et al. Mature cystic teratoma of the ovary with struma ovarii and benign Brenner tumor: a case report with immunohistochemical characterization. Int $\mathrm{J}$ Gynecol Pathol. 2002,21:74-77.

[5] Nogales F, Talerman A, Kubick-Huch RA, et al. Germ cell tumor. In: Tavassoli FA, Devilee P, editors. World Health Organization Classification of Tumours. Pathology and genetics of tumors of the breast and female genital organs. Lyon: IARC Press; 2003. pp. 163-175.

[6] Terada T, Kawaguchi M. Primary clear cell adenocarcinoma of the peritoneum. Tohoku J Exp Med. 2005;206:271-275.

[7] Nasioudis D, Sisti G, Holcomb K.,et al.Malignant Brenner tumors of the ovary; a population-based analysis. Gynecol. Oncol. 2016:142, 44-49.

[8] Han JH, Kim DY, Lee SW, et al. Intensive systemic chemotherapy is effective against recurrent malignant Brenner tumor of the ovary: An analysis of 10 cases within a single center. Taiwan J Obstet Gynecol, 2015, 54(2): 178-182.

[9] Roma AA, Masand RP. Ovarian Brenner tumors and Walthard nests: a histologic and immunohistochemical study. Hum Pathol. 2014; 45: 2417-22.

[10] Takeuchi M, Matsuzaki K, Sano N, et a1. Malignant Brenner tumor with transition from benign to malignant components: computed tomographic and magnetic resonance imaging findings with pathological correlation. J Comput Assist Tomogr, 2008, 32(4): 553-554.

[11] Shevchuk MM, Fenoglio CM, Richart RM. Histogenesis of Brenner tumors, I: histology and ultrastructure. Cancer. 1980;46:2607-16. 
[12] Quan J, Jin L, Hu J,et al. Brenner tumor of the testis: a case report and review of the literature. Mol Clin Oncol. 2017;6:119-21.

[13] Gedikbasi A, Ulker V, Aydin O, et al. Brenner tumor in pregnancy: clinical approach and pathological fifindings. Obstet Gynaecol Res, 2009, 35(3): 565-568.

[14] Kondi-Pafifiti A,Kairi-Vassilatou E,Iavazzo C.Clinico-pathological features and immuneo-profile of 30 cases of Brenner ovarian tumors. Arch Gynecol Obstet, 2012, 285(6): 1699-1702.

[15] Hermanns B, Faridi A, Rath W, et al. Differential diagnosis, prognostic factors, and clinical treatment of proliferative Brenner tumor of the ovary. Ultrastruct Pathol. 2000 May-Jun;24(3):191-6.

[16] Turgay B, Koyuncu K, Taşkın S,et al. Features of ovarian Brenner tumors: Experience of a single tertiary center. Turk J Obstet Gynecol. 2017 Jun;14(2):133-137. 


\section{Figures}

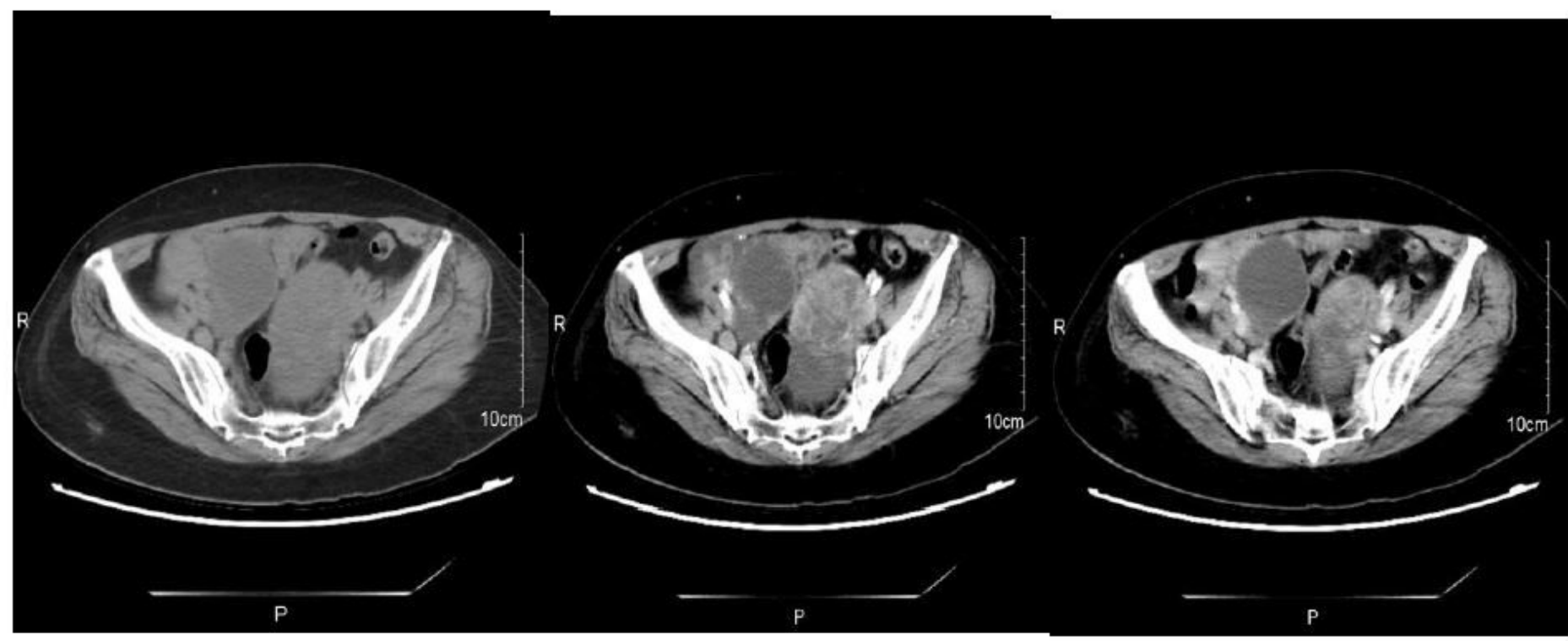

Arterial phase

Diagram of plain

venous phase diagram

\section{Figure 1}

Abdominal CT of the patient (Enhanced CT scan of the whole abdomen, arterial phase, venous phase) Diagnosis of CT:1. Bilateral adnexal cystic and the solid mass-the possibility of ovarian cystadenocarcinoma. 2. The irregular shape of the cervix. 3. Small amounts of pelvic fluid. 4. Multiple small retroperitoneal lymph nodes.

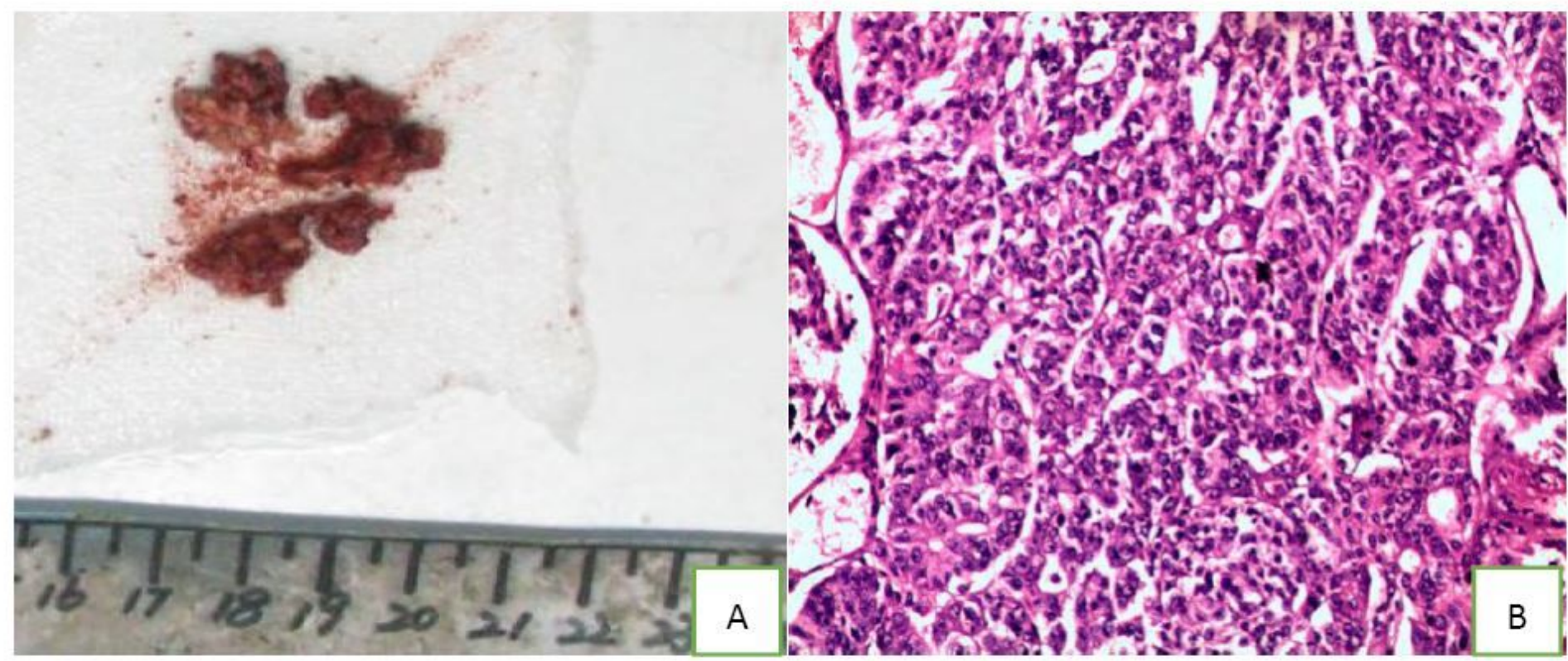

Figure 2 
Poorly differentiated squamous cell carcinomam of the cervix.

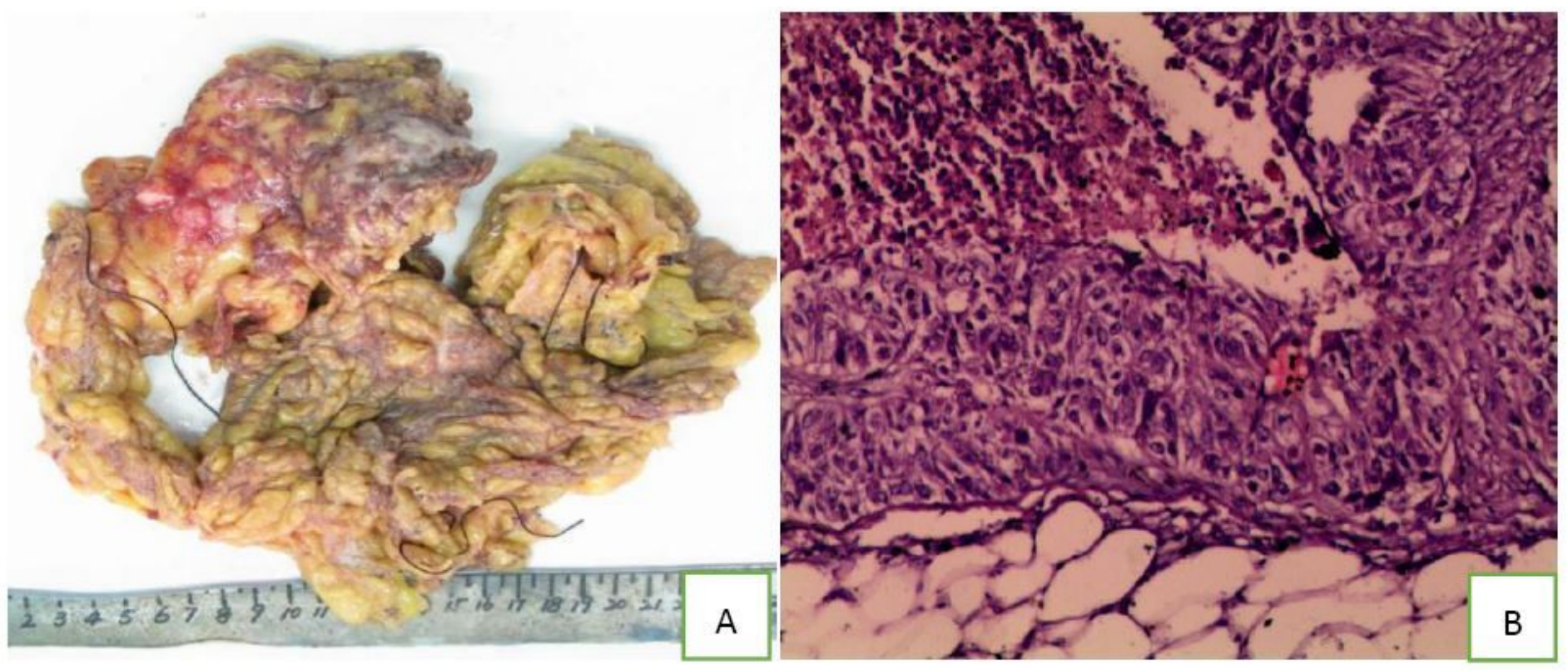

\section{Figure 3}

The omentum majus thickening to form an $8 \mathrm{~cm}$ x $8 \mathrm{~cm}$ hard cake. Multifocal, implantable, carcinoma nodules of the omentum majus

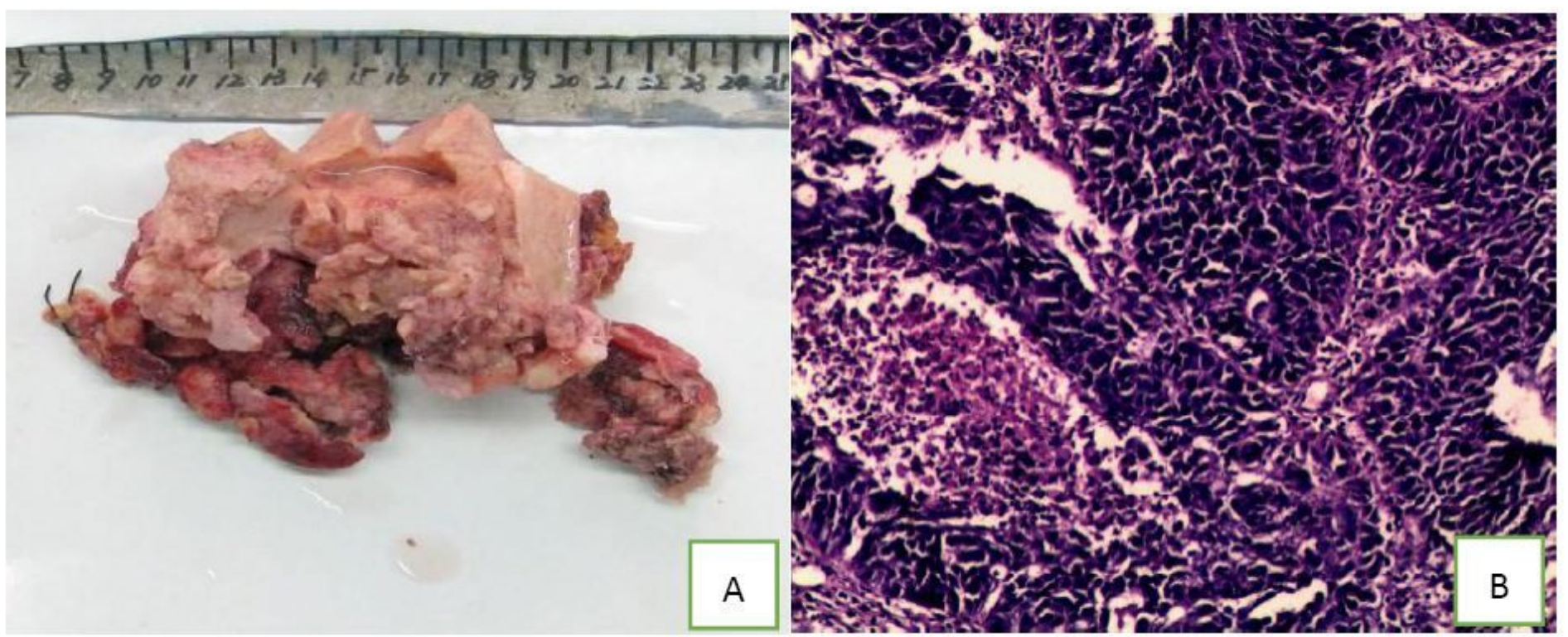

\section{Figure 4}

Specimen of the Operation(A and B) A: Bilateral malignant Brenner ovarian tumor extending to the entire outer Mesangium with multiple foci of necrosis and cystic degeneration. B: Both fallopian tubes and their mesentery are infiltrative, with multiple nodules surrounding the double appendages. Carcinoma cells 
invading the uterine wall and reaching the uterine cavity and internal orifice. No invasive carcinoma found in the external orifice of the cervix.

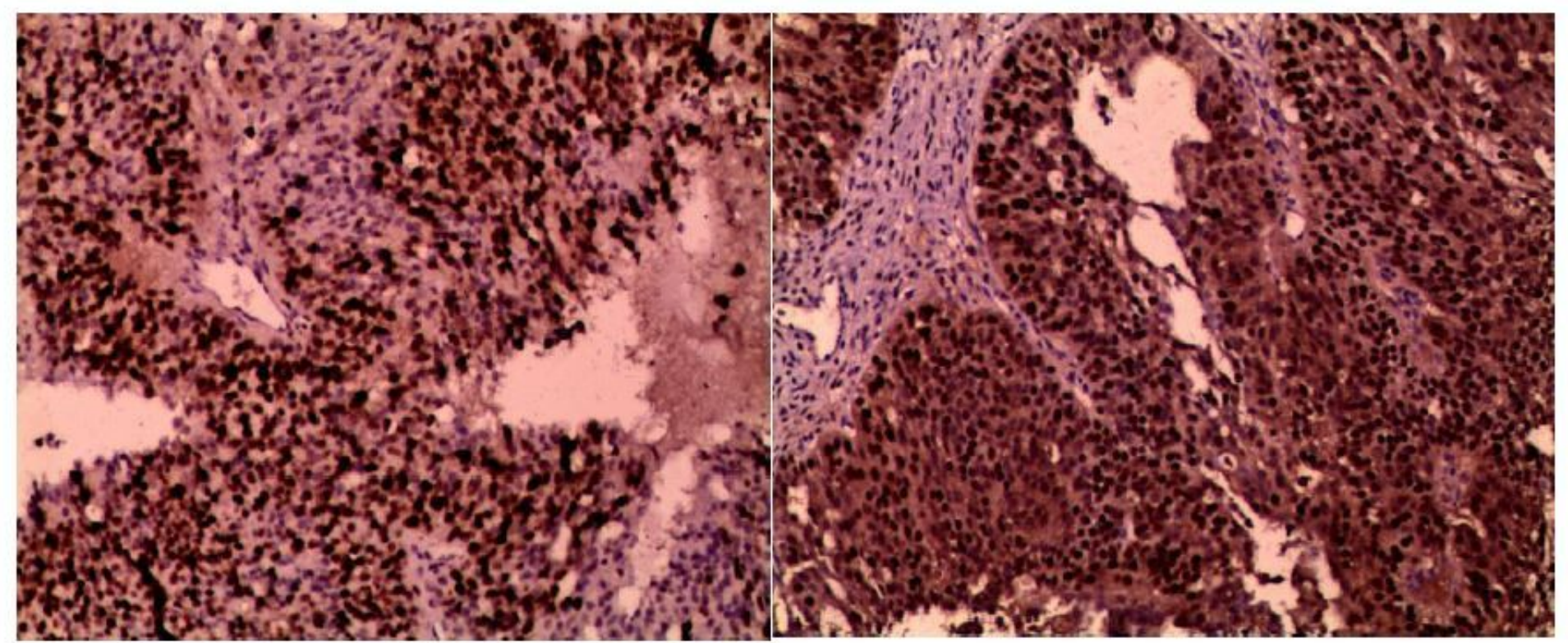

\section{Figure 5}

Histopathological characteristics of the specimen by HE staining (HEx100) IHC immunohistochemical results: CK5/6(multiple foci + ) , CK34 E12(multiple foci +) , P63(multiple foci +) , CK20(multiple foci +) , CA125(+) , PR (95\%) , ER (80\%) , PGP (++) , P53(++) , Ki-67(+ about 80\%) . Combined with HE staining, it was indicated to be malignant ovarian Brenner tumor, and the performance of most cells was corresponded with transitional cell carcinoma, the performance of a small number with squamous cell carcinoma and undifferentiated carcinoma with high proliferative activity. 


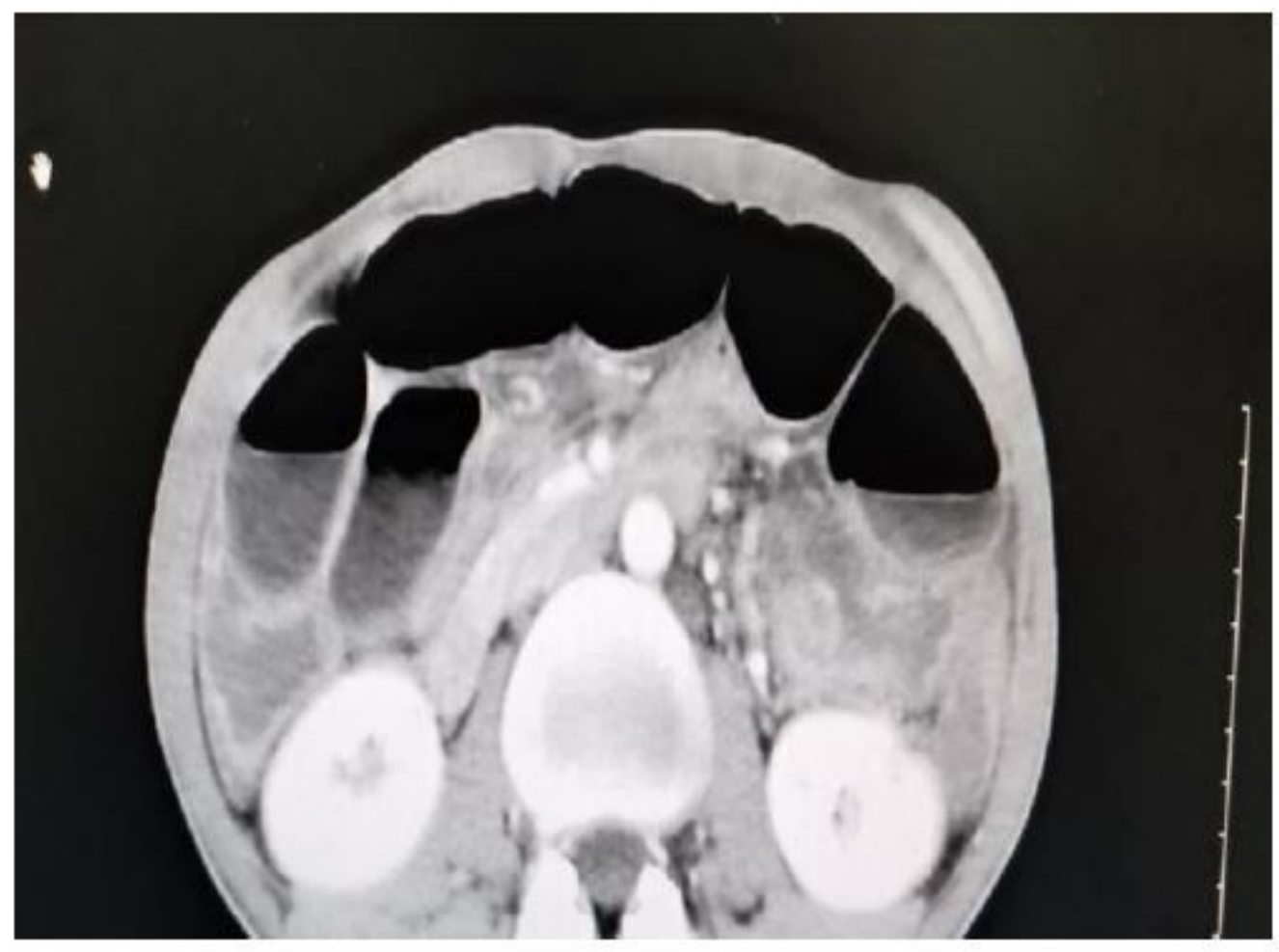

Figure 6

Abdominal CT of the patient (Enhanced CT scan of the whole abdomen, arterial phase, axial) 